Check for updates

Cite this: J. Mater. Chem. B, 2020 8, 9084

Received 26th April 2020,

Accepted 19th August 2020

DOI: 10.1039/d0tb01089h

rsc.li/materials-b

\title{
Photonic hyperthermal and sonodynamic nanotherapy targeting oral squamous cell carcinoma $\dagger$
}

\author{
Jiaxin Zuo, ${ }^{a}$ Minfeng Huo, ${ }^{* b}$ Liying Wang, ${ }^{b}$ Jia Li, ${ }^{a}$ Yu Chen $\left(\mathbb{D}{ }^{* c}\right.$ and \\ Ping Xiong (iD) *a
}

\begin{abstract}
Nanomedicine that enables multiple synergetic treatments provides effective non-invasive treatment modalities for cancer therapy. Yet treatments for oral squamous cell carcinoma (OSCC) are rarely reported. Here, we designed OSCC-targeting multi-functional nanomedicines to overcome the therapeutic obstacles during OSCC treatments, including ineffective chemotherapy, and the traumatic surgery and radiotherapy. The urokinase plasminogen activator receptor (UPAR)-targeting ligand AE105 decorated dendritic mesoporous silica nanoparticles (DMSN) encapsulating photonic active ultrasmall $\mathrm{Cu}_{2-x} \mathrm{~S}$ NPs and sonosensitizer Rose Bengal (RB) have been rationally designed and constructed (designated as $\mathrm{Cu}_{2-x} \mathrm{~S}-\mathrm{RB}(\mathrm{DDMSN}-\mathrm{AE105}$, abbreviated as CRDA). These CRDAs initially target the uPAR, which is overexpressed in the OSCC cell membrane, to increase the localized accumulation of CRDAs at tumor sites. Under the irradiation of both near-infrared laser and ultrasound, the in situ photonichyperthermal and sonodynamic effects are respectively enabled to induce the cell death of OSCC. Upon both in vitro/in vivo challenges, tumor cells/xenografts have been efficiently eradicated, achieving the targeting and synergetic treatment modality against the OSCC with satisfactory biocompatibility.
\end{abstract}

\section{Introduction}

The application of "nanomedicine" against cancer is one of the medical research favorites at present. Various nanoplatforms have been constructed using unique theranostic functionalities to meet sophisticated biomedical circumstances. ${ }^{1-4}$ In particular, multifunctional nanoparticles (NPs) combined with various therapeutic modalities, including photonic hyperthermia, photodynamic, ${ }^{1}$ chemotherapy, ${ }^{5}$ magnetotherapy ${ }^{6}$ and sonodynamic therapy, ${ }^{7,8}$ can generate multiple theranostic modalities in practical use to achieve a synergistic therapeutic outcome. Among diverse nanoplatforms, the biomedical applications of

\footnotetext{
${ }^{a}$ Department of Ultrasound, Shanghai Ninth People's Hospital, Shanghai Jiaotong University School of Medicine, Shanghai, 200011, P. R. China.

E-mail: xiongp1281@sh9hospital.org.cn

${ }^{b}$ State Key Laboratory of High Performance Ceramics and Superfine Microstructure, Shanghai Institute of Ceramics, Chinese Academy of Sciences, Shanghai, 200050, P. R. China. E-mail: mfhuo@mail.sic.ac.cn

${ }^{c}$ School of Life Sciences, Shanghai University, Shanghai, 200444, P. R. China. E-mail: chenyuedu@shu.edu.cn

$\dagger$ Electronic supplementary information (ESI) available: Supplementary figures contain STEM image and dark-field images of $\mathrm{Cu}_{2-x}$ S NPs, STEM image and SEM image of DMSNs and CRDAs, XRD pattern of $\mathrm{Cu}_{2-x} \mathrm{~S}$ NPs, UV-vis absorption spectra of CRDs, CRDAs and $\mathrm{Cu}_{2-x} \mathrm{~S} @ D$ DMSN-AE105 NPs, UV-vis absorption spectra of varied RB doses and the corresponding dose-absorption standard curve. See DOI: 10.1039/d0tb01089h
}

mesoporous silica nanoparticles (MSNs) have received considerable attention during the past decades. ${ }^{9-12}$ MSNs feature a well-defined mesoporous structure that can not only encapsulate therapeutic drugs but also functional nanoparticles to support the construction of multifunctional nanoplatforms. This progress provides an important basis for the clinical transformation of nanomedicine with appealing therapeutic potentials.

Oral squamous cell carcinoma (OSCC) is the leading cause of death in head and neck squamous cell carcinoma (HNSCC) with high malignancy, easy metastasis, and high recurrence. ${ }^{13,14}$ One of the reasons for the poor prognosis of OSCC-patients is the inadequate therapeutic efficacy for complete OSCC destruction. ${ }^{13,15}$ The most frequently adopted treatments are surgery supplemented with radiotherapy and/or chemotherapy. Surgical dissection is associated with considerable trauma and serious side-impacts, which substantially affect the post-treatment life quality of the patients. ${ }^{15-17}$ Therefore, applications of nanomedicine with great medical significance for the treatment of OSCC can effectively ameliorate the prognosis. Photonic hyperthermal therapy (PTT) typically refers to the use of near-infrared light to enable photonic hyperthermia in the presence of photonic active agents. These agents such as two-dimensional nanosheets, ${ }^{18,19}$ organic semiconductors, ${ }^{20}$ and other inorganic metal NPs (e.g., gold $\mathrm{NPs},{ }^{21-23} \mathrm{CuS}^{24,25}$ and $\mathrm{Fe}_{3} \mathrm{O}_{4}{ }^{26}$ ) can convert incident light energy 
into heat for tumor destruction. Among these nanoparticulate photonic active agents, ultrasmall $\mathrm{Cu}_{2-x} \mathrm{~S}$ NPs $(\sim 5 \mathrm{~nm})$ feature high therapeutic prospects. ${ }^{27}$ Although it has been demonstrated that PTT could generate a prominent therapeutic effect against tumors, yet the uneven distribution of heat and poor tissue penetration depth enabled by the laser during treatment may not possibly lead to complete tumor eradiation, ultimately resulting in tumor reoccurrence. ${ }^{28,29}$ In order to guarantee a therapeutic outcome and satisfactory prognosis, enhanced tumor-site accumulations of therapeutic nanoagents are urgently demanded, specifically by targeting engineering, which can simultaneously reduce the side impacts while improving the therapeutic outcome.

The present research in the field of nanomedicine for OSCC treatment is mostly focused on PTT with chemotherapy. ${ }^{30,31}$ Statistical data have shown that, over the past 40 years, the application of cytotoxic chemotherapeutic drugs has not significantly ameliorated the survival or life quality of patients with oral cancer. ${ }^{32,33}$ Therefore, exploring new synergistic therapeutic modalities to achieve safe and effective treatment for OSCC, as well as to ameliorate the prognosis, is highly urgent and necessary. Reactive oxygen species (ROS) play critical roles in cell apoptosis, ${ }^{7}$ which could be generated by sonodynamic therapy (SDT). ${ }^{29}$ Recently, ultrasound (US)-triggered SDT was found to be promising for practical use because of its noninvasive nature and high tissue-penetrating capability. ${ }^{29,34}$ For instance, McEwan et al. designed RB containing sonodynamic nanoplatforms PLGA MB-RB, ${ }^{35} \mathrm{Oxy} \mathrm{MB}+\mathrm{RB}^{36}$ and realized satisfactory SDT performance with a minimal $\mathrm{RB}$ dose of as low as $1 \mu \mathrm{M}$. Therefore, the elaborate combination of laser-triggered photonic hyperthermia and US-activated sonodynamic treatment is expected to achieve highly efficient OSCC treatment outcomes.

It has been documented that epidermal growth factor receptors (EGFRs) are overexpressed in OSCC, and the inhibition of the EGFRs has been a desirable therapeutic target for OSCC treatment. Currently, cetuximab is the only EGFR-targeting drug approved by the US Food and Drug Administration. However, secondary drug resistance would inevitably occur during the medication. ${ }^{37}$ Recently, another OSCC-targeting site, urokinase plasminogen activator receptor (UPAR) has been discovered. The over-expression of UPAR in the OSCC cell membrane can reinforce the signal transmission of the UPARintegrin pathway, a critical transmembrane pathway for OSCC metastasis and proliferation. ${ }^{38-40}$ At the same time, uPAR exhibits a higher OSCC specificity when compared to EGFR. Therefore, UPAR can be used as an alternative biocompatible target against OSCC with better performance.

In this work, we report uPAR-targeting PTT/SDT synergistic therapeutic $\mathrm{Cu}_{2-x}$-RB@DMSN-AE105 (abbreviated as CRDA) nanoplatforms for targeted PTT/SDT synergistic therapy of OSCC. As the drug delivery system, biocompatible dendritic mesoporous silica NPs (DMSN) can biodegrade slowly in the acidic microenvironment of the tumor, ${ }^{9}$ achieving the cargo release. DMSNs were employed to encapsulate photonic active $\mathrm{Cu}_{2-x} \mathrm{~S} \mathrm{NPs}^{27}$ and sonosensitive Rose Bengal (RB) ${ }^{41}$ to realize synergistic PTT/SDT nanotherapeutics. For OSCC targeting design, AE105 (H-Asp-Cha-Phe-(d)Ser-(d)Arg-Tyr-Leu-Trp-Ser$\mathrm{OH})$ peptide features prominent targeting against uPAR that originates from the competing binding towards UPAR. $^{42-44}$ When modified on the surface of DMSNs, OSCC targeting of CRDAs can be additionally achieved, providing an intriguing treatment modality against OSCC with targeting and synergistic performance.

\section{Materials and methods}

\section{Materials}

Cetyltrimethylammonium chloride (CTAC, Sigma-Aldrich Co.), tetraethyl orthosilicate (TEOS, Sinopharm Chemical Reagent Co.), triethanolamine (TEA, Sinopharm Chemical Reagent Co.), sodium salicylate (NaSal, Sigma-Aldrich Co.), hydrochloric acid (Sinopharm Chemical Reagent Co.), sulfur powder (Aladdin Biochemical Technology Co., Ltd), oleylamine (Aladdin Biochemical Technology Co., Ltd), chloroform (Sinopharm Chemical Reagent Co.), silane-PEG-carboxyl (silane- $\mathrm{PEG}_{2000}-\mathrm{COOH}$, Shanghai Ponsure Biotech, lnc.), AE105 peptide (D-ChaFsrYLWS, Nanjing Peptide Biotechnology Co., Ltd), Red Bengal (RB, Aladdin Biochemical Technology Co., Ltd).

\section{Synthesis of DMSNs}

In a typical procedure, TEA $(0.068 \mathrm{~g})$ was initially mixed with deionized water $(5 \mathrm{~mL})$. Then, $0.5 \mathrm{~mL}$ of the above solution was mixed with $24.5 \mathrm{~mL}$ of deionized water, and placed in a water bath with a magnetic stirrer (300 rpm at $80{ }^{\circ} \mathrm{C}$ for $\left.30 \mathrm{~min}\right)$. CTAC (0.38 g), as the structure-directing agent, was added to the solution. After complete dissolution, NaSal $(0.168 \mathrm{~g})$ was added to the solution and the reaction was allowed to continue for $1 \mathrm{~h}$, followed by the dropwise addition of TEOS $(2 \mathrm{~mL})$. After another $2 \mathrm{~h}$, the product was collected by centrifugation. CTAC was removed using a mixture of ethanol and concentrated $\mathrm{HCl}$ (6\%) by extracting for $24 \mathrm{~h}$ at $70{ }^{\circ} \mathrm{C}$ three times. Finally, the product was collected by centrifugation, washed with water several times, and dispersed in $20 \mathrm{~mL}$ of ethanol.

\section{Synthesis of ultrasmall $\mathrm{Cu}_{2-x} \mathrm{~S}$ NPs}

$\mathrm{Cu}_{2-x} \mathrm{~S}$ NPs with diameter $2-5 \mathrm{~nm}$ were synthesized according to the reported literature. ${ }^{27}$ Initially, sulfur $(64 \mathrm{mg}$ ) was immersed in oleylamine $(12 \mathrm{~mL})$ and placed in a water bath at $70{ }^{\circ} \mathrm{C}$ with a magnetic stirrer until a dark red-brown solution was formed. A chloroform solution $(40 \mathrm{~mL})$ containing copper acetylacetonate (523.5 $\mathrm{mg})$ and oleylamine $(10 \mathrm{~mL})$ was added to the above solution in a dropwise manner. During the reaction of $0.5 \mathrm{~h}$ at $70{ }^{\circ} \mathrm{C}$, the mixture turned from brown-yellow to dark-green. Ethanol was added to precipitate the reaction. Finally, the product was collected by centrifugation, washed with water several times, and re-dispersed in $20 \mathrm{~mL}$ of chloroform.

\section{Fabrication of CRDAs}

First, $2 \mathrm{~mL}$ of $\mathrm{Cu}_{2-x} \mathrm{~S}$ NPs dispersed in chloroform $\left(10 \mathrm{mg} \mathrm{mL}{ }^{-1}\right)$ was added to $2 \mathrm{~mL}$ of DMSNs dispersed in chloroform $\left(5 \mathrm{mg} \mathrm{mL}^{-1}\right)$ with agitation. After $12 \mathrm{~h}$ of encapsulation, $\mathrm{Cu}_{2-x} \mathrm{~S} @ D M S N$ NPs 
were formed and collected by centrifugation. Then, $\mathrm{Cu}_{2-x} \mathrm{~S} @$ DMSN NPs $(20 \mathrm{mg})$ were added to $10 \mathrm{~mL}$ ethanol containing $10 \mathrm{mg} \mathrm{mL}{ }^{-1}$ of silane-PEG-NH ${ }_{2}($ molecular weight $=2000)$ at $60{ }^{\circ} \mathrm{C}$ with magnetic stirring. After $24 \mathrm{~h}$ of PEGylation, the PEGylated $\mathrm{Cu}_{2-x} \mathrm{~S} @ D$ MSN NPs were centrifuged and washed several times to remove residual PEG. Decoration of AE105 onto DMSNs was accomplished by the reaction between AE105 and PEGylated $\mathrm{Cu}_{2-x} \mathrm{~S} @ D$ DSN NPs in MES solution in the presence of EDC and NHS for $12 \mathrm{~h}$. After the centrifugation and purification process, $\mathrm{Cu}_{2-x} \mathrm{~S} @$ DMSN-AE105 NPs were collected. $2 \mathrm{~mL}$ of $\mathrm{Cu}_{2-x} \mathrm{~S} @ D M S N-A E 105$ NPs re-suspended in ethanol $\left(1 \mathrm{mg} \mathrm{mL}{ }^{-1}\right.$ ) was mixed with $\mathrm{RB}(4 \mathrm{mg})$ for $12 \mathrm{~h}$ to encapsulate the RB sonosensitizer. Finally, the $\mathrm{Cu}_{2-x} \mathrm{~S}-\mathrm{RB} @ D M S N-A E 105$ NPs (CRDAs) were obtained. To produce $\mathrm{Cu}_{2-x} \mathrm{~S}-\mathrm{RB} @ D M S N$ NPs (CRDs), 2 mL of PEGylated $\mathrm{Cu}_{2-x} \mathrm{~S} @ D M S N$ NPs in ethanol $\left(1 \mathrm{mg} \mathrm{mL}{ }^{-1}\right)$ was mixed with $\mathrm{RB}(4 \mathrm{mg})$. After an additional $12 \mathrm{~h}$ of encapsulation, the CRDs were collected by centrifugation.

To explore the release profile of $\mathrm{RB}, 10 \mathrm{~mL}$ PBS solutions at $\mathrm{pH}$ 7.4 and 5.5, respectively, containing CRDAs (RB: $10 \mu \mathrm{g} \mathrm{mL}^{-1}$ ), were stirred on a magnetic stirrer. At pre-determined time points $(0.5,1,2,4,8,12,24 \mathrm{~h})$, aliquots of the solution were centrifuged and the supernatant was used for UV-vis measurements.

\section{Characterization}

Transmission electron microscopy (TEM) images, energydispersive X-ray spectroscopy (EDS) and corresponding EDSmapping were collected on a JEM-2100F transmission electron microscope operated at $300 \mathrm{kV}$. DLS was conducted on a Nano ZS90 Zetasizer (Malvern Instruments, Malvern, UK). UV-vis-NIR absorption spectra were recorded on a UV-3600 Shimadzu spectrometer. Zeta potential was tested on a Zetasizer Nanoseries instrument (Nano ZS90). The nitrogen $\left(\mathrm{N}_{2}\right)$ adsorptiondesorption isothermal curve and the corresponding pore-size distribution of DMSNs and $\mathrm{Cu}_{2-x} \mathrm{~S} @ D$ MSN NPs were calculated using Micromeritics Tristar 3000 system. Inductively coupled plasma atomic emission spectrometry (ICP-AES) was conducted on a VISTA instrument (Varian Company, American). ESR characterization was performed on a Bruker EMX electron paramagnetic resonance (EPR) spectrometer.

\section{Sonodynamic and photonic hyperthermia performance of CRDs}

In this study, DPBF was employed to evaluate the singlet oxygen $\left({ }^{1} \mathrm{O}_{2}\right)$ generation induced by $\mathrm{RB}$ and CRDs respectively upon US irradiation. Briefly, RB or CRDs (RB: $10 \mu \mathrm{g} \mathrm{mL}^{-1}$ ) was mixed with DPBF (50 $\mu \mathrm{M}$ in DMF), diluted with ethanol. Then, the above solution was exposed to US irradiation $\left(1 \mathrm{MHz}, 2.0 \mathrm{~W} \mathrm{~cm}^{-2}, 50 \%\right.$ duty cycle, $2 \mathrm{~min}$ ) in the dark. The absorbance of the solution was then recorded on a UV-vis spectrometer.

For ESR assays, triacetonediamine (TEMP, Aladdin Biochemical Technology Co., Ltd) was used to trap ${ }^{1} \mathrm{O}_{2}$ generated by $\mathrm{RB}$ and CRDAs using an ultrasound trigger. Typically, dimethyl formamide (DMF) solution containing $\mathrm{RB}$ and CRDAs (RB: $\left.8 \mu \mathrm{g} \mathrm{mL}{ }^{-1}\right)$ were exposed to US irradiation $\left(1.0 \mathrm{MHz}, 2.0 \mathrm{~W} \mathrm{~cm}^{-2}\right.$, $50 \%$ duty cycle, $2 \mathrm{~min})$ in the presence of TEMP $(20 \mu \mathrm{M})$.
Aliquots of the assay solution were then transferred into the quartz tube for ESR measurements.

For the assessment of the photonic hyperthermia performance of CRDs, the localized temperature elevation profile of CRDs $\left(0,25,50,100\right.$ and $\left.200 \mu \mathrm{g} \mathrm{mL}^{-1}\right)$ under NIR laser irradiation (Shanghai Connect Fiber Optics, $1064 \mathrm{~nm}, 1.5 \mathrm{~W} \mathrm{~cm}$, $5 \mathrm{~min}$ ) was recorded on an infrared thermal imaging camera (A325SC; FLIR Systems, Wilsonville, OR, USA). The photonic hyperthermia stability of CRDs was revealed by the negligible fluctuations of the maximum temperature during five sequential laser on/off cycles against CRDs (400 $\mu \mathrm{g} \mathrm{mL} \mathrm{mL}^{-1}, 1064 \mathrm{~nm}$, $\left.1.5 \mathrm{~W} \mathrm{~cm}^{-2}, 5 \mathrm{~min}\right)$.

\section{In vitro synergetic performance of PTT/SDT of CRDs against OSC-19 cells}

OSC-19 cells were grown in DMEM with 10\% FBS and 1\% penicillin/streptomycin in an incubator at $37{ }^{\circ} \mathrm{C}$ in an atmosphere of $5 \% \mathrm{CO}_{2}$. Cells were initially seeded into 96 -well plates with a density of $10^{4}$ cells per well for $24 \mathrm{~h}$. CRDs $(0,25,50,100$,

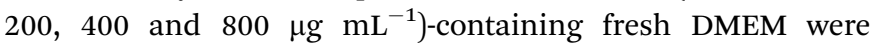
replaced for further co-incubations. For cytocompatibility investigations, medium was discarded $24 \mathrm{~h}$ or $48 \mathrm{~h}$ post-incubation and cells were rinsed with PBS. Cell viability was performed using cell-counting kit-8 (CCK-8) according to the protocols.

For in vitro PTT/SDT therapeutics, cells co-cultured with CRDs $\left(0,25,50,100,200\right.$ and $\left.400 \mu \mathrm{g} \mathrm{mL}{ }^{-1}\right)$ for $4 \mathrm{~h}$ were divided into three groups according to the respective treatments: US group (US irradiation); laser group (laser irradiation); US + laser group (US irradiation, followed by laser irradiation). The parameters of US irradiation were $1.0 \mathrm{MHz}, 2.0 \mathrm{~W} \mathrm{~cm}^{-2}, 50 \%$ duty cycle, $2 \mathrm{~min}$. The parameters of laser irradiation were $1064 \mathrm{~nm}, 1.5 \mathrm{~W} \mathrm{~cm}^{-2}$, $5 \mathrm{~min}$. Then, the medium was discarded and cells were rinsed with PBS several times. Cell viability was determined using a cellcounting kit-8 (CCK-8) according to the protocols.

\section{In vitro synergetic performance of PTT/SDT of CRDs against OSC-19 cells by confocal laser scanning microscopy (CLSM)}

Cells were initially seeded into confocal disks for $12 \mathrm{~h}$ with a density of $10^{5}$ cells per disk. Then, these disks were divided into seven groups for respective treatments: control group (untreated); US (US irradiation only); laser (laser irradiation only); NPs group (co-incubation with CRDs for $4 \mathrm{~h}$ ); CRDs + laser group (laser irradiation followed by co-incubation of CRDs for $4 \mathrm{~h}$ ); CRDs + US group (US irradiation followed by co-incubation of CRDs for $4 \mathrm{~h}$ ); $\mathrm{NP}+\mathrm{US}+$ laser group (coincubation of CRDs for $4 \mathrm{~h}$ with subsequent US irradiation and laser irradiation). CRDs: $100 \mu \mathrm{g} \mathrm{mL} \mathrm{m}^{-1}$, laser: $1064 \mathrm{~nm}$, $1.5 \mathrm{~W} \mathrm{~cm}^{-2}, 5 \mathrm{~min}$, US: $1.0 \mathrm{MHz}, 2.0 \mathrm{~W} \mathrm{~cm}^{-2}, 50 \%$ duty cycle, $2 \mathrm{~min}$. Then, the cell culture medium was replaced with nonfluorescent DCFH-DA solution and incubated for $30 \mathrm{~min}$. Finally, cells were washed gently with PBS and observed using CLSM on a FV1000 system (Olympus, Tokyo, Japan).

For visualization of live/dead OSC-19 cells by CLSM, OSC-19 cells were stained by calcein-AM/PI (calcein-AM $(40 \mu \mathrm{M})$ and PI $(60 \mu \mathrm{M})$ in PBS). After $10 \mathrm{~min}$ of staining, cells were washed thrice with PBS prior to CLSM observation. 


\section{In vivo synergetic therapeutics of CRDAs against OSCC xenografts in mice}

Procedures involving animal experiments were in accordance with the guidelines set by the Animal Care and Use Committee of Shanghai Ninth People's Hospital within the School of Medicine of Shanghai Jiao Tong University (Shanghai, China). Healthy male BALB/c mice ( 4 weeks) were purchased by Shanghai XipuerBeikai Experimental Animals (Shanghai, China). They were randomly divided into four groups ( $n=4$ in each group) followed by intravenous injection of CRDAs at elevated doses $\left(0 \mathrm{mg} \mathrm{kg}{ }^{-1}\right.$ (control), $5 \mathrm{mg} \mathrm{kg}^{-1}, 10 \mathrm{mg} \mathrm{kg} \mathrm{kg}^{-1}$, and $20 \mathrm{mg} \mathrm{kg}^{-1}$ ) to assess in vivo biocompatibility. These mice were sacrificed after 15 days of feeding for whole blood panel tests, biochemical analyses and H\&E-stained organ slices.

For in vivo biodistribution analysis, 8 healthy male BALB/c nude mice were divided into 2 groups $(n=4)$ randomly. These mice were then injected with OSC-19 cells $\left(10^{6}\right.$ cells per site, subcutaneous injection (s.c.)) in the right hind limb to establish OSCC-xenograft models. When the average volume of the tumors exceeded $150 \mathrm{~mm}^{3}$, mice were injected intravenously (i.v.) with CRDAs or CRDs (20 mg kg ${ }^{-1}$ ) in PBS for each group respectively. At predetermined time-points, mice were sacrificed at 4, 8, 12 and $24 \mathrm{~h}$ post-injection. Dissected organs and tumors were weighted and homogenized, followed by ICP-AES analysis to determine the concentration of $\mathrm{Cu}$. The distribution of CRDAs/CRDs in organs and the tumor-targeting efficiency were calculated as the species concentration. For in vivo tumor-targeting analyses, in vivo photoacoustic imaging was performed on two male BALB/c nude mice bearing an OSCC xenograft on the right hind limb. When the volume of the tumors exceeded $50 \mathrm{~mm}^{3}$, mice were injected intravenously (i.v.) with CRDAs or CRDs (20 mg kg-1) in PBS for each group respectively. At predetermined time-points $(0,4,8,12$ and $16 \mathrm{~h}$ post-injection), photoacoustic imaging of the nude mice was performed.

For in vivo PTT/SDT therapeutics, 42 OSCC-bearing mice were randomly divided into 7 groups ( $n=6$ in each group): control group: i.v. injection of saline; US group: i.v. injection of saline, followed by US irradiation; laser group: i.v. injection of saline, followed by laser irradiation; CRDAs group: i.v. injection of CRDAs; CRDAs + laser group: i.v. injection of CRDAs, followed by laser irradiation; CRDAs + US group: i.v. injection of CRDAs, followed by US irradiation; CRDAs + US + laser group: i.v. injection of CRDAs, followed by sequential US irradiation and laser irradiation. Treatment parameters: injection doses of CRDAs are $10 \mathrm{mg} \mathrm{kg}^{-1}$ (laser: $1064 \mathrm{~nm}, 1.5 \mathrm{~W} \mathrm{~cm}{ }^{-2}, 5 \mathrm{~min}$;

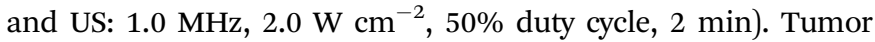
tissues were collected from one mouse in each group at $24 \mathrm{~h}$ after treatment for ultrathin section preparation and histopathological staining using H\&E, TUNEL, and antigen Ki-67 respectively. After respective treatment, mice were weighed and the tumor sizes of mice in different groups were recorded every other day during the 15 days evaluation period. The length $(L)$ and width $(W)$ of tumors were measured and the tumor volume $(V)$ was calculated according to the following formula:

$$
V=L \times W^{2} / 2
$$

To evaluate the survival rate of the mice, the number of the living mice was recorded every other day. Following the guidelines of the animal experiments, when the tumor volume exceeded $1000 \mathrm{~mm}^{3}$, the mice were sacrificed. All the mice were sacrificed after the two-month evaluation period.

\section{Statistical analyses}

Data are the mean \pm standard deviation. Significance of the data was obtained according to the Student's $t$-test. $P<0.05$ was considered as significant.

\section{Results and discussion}

\section{Design, synthesis, and characterization of CRDAs}

The design and synthesis of CRDAs are illustrated in Fig. 1a. DMSNs and $\mathrm{Cu}_{2-x} \mathrm{~S}$ NPs were initially fabricated respectively. Featuring large mesopores of DMSNs, the $\mathrm{Cu}_{2-x} \mathrm{~S}$ NPs and RB could be enabled to be sequentially encapsulated. Further decoration of the uPAR-targeting ligand AE105 leads to the fabrication of CRDAs, which guarantee the targeting biosafety and synergistic therapeutic performance of CRDAs for efficient tumor therapy both in vitro and in vivo.

After intravenous injection, the CRDAs could accumulate into tumor tissue for nanotherapies (Fig. 1b). Typically, CRDAs could penetrate into tumor tissue via the enhanced permeability and
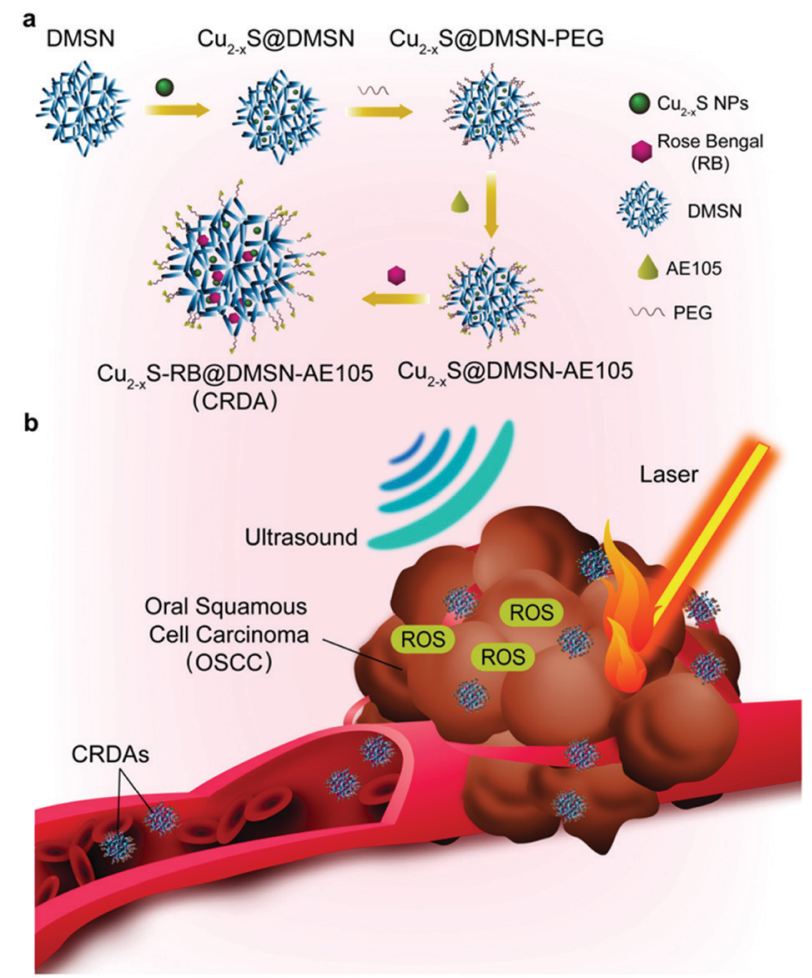

Fig. 1 Schematic diagram of the synthesis of CRDAs in combating OSCC. (a) The synthetic route for $\mathrm{Cu}_{2-x} \mathrm{~S} N P s$ and RB encapsulated, AE105 decorating CRDAs. (b) Therapeutic modalities of CRDAs for targeted photonic hyperthermal/sonodynamic therapy against OSCC. CRDAs can target and accumulate in the UPAR-overexpressed OSCC tumor sites to produce synergistic PTT/SDT therapeutics upon NIR laser and US irradiations. 
retention (EPR) effect and the active targeting by uPAR-targeting ligand AE105. Then, the $\mathrm{Cu}_{2-x} \mathrm{~S}$ NPs of CRDAs could generate the photonic hyperthermal effect for localized hyperthermia against OSCC under laser irradiation at $1064 \mathrm{~nm}$, while the sonosensitive $\mathrm{RB}$ could induce the sonodynamic effect to produce singlet oxygen species under US irradiation. With these prominent effects, the OSCC cells/tissues are considered to be completely eradicated.

Transmission electron microscopy (TEM) and dynamic light scattering (DLS) were employed to characterize the morphology, size and composition of $\mathrm{Cu}_{2-x} \mathrm{~S}$ NPs, DMSNs, and CRDAs respectively (Fig. 2). Typically, $\mathrm{Cu}_{2-x} \mathrm{~S}$ NPs with a size of $2-5 \mathrm{~nm}$ were synthesized via the pyrolysis method. ${ }^{27}$ The synthetic $\mathrm{Cu}_{2-x} \mathrm{~S}$ are hydrophobic NPs coated with an oleylamine layer (Fig. 2a and Fig. S1a, b, ESI $\dagger$ ). DMSNs with a size of approximately $100 \mathrm{~nm}$ were characterized with dendritic mesoporous structures that were suitable for the encapsulation of $\mathrm{Cu}_{2-x} \mathrm{~S}$ NPs and RB, as confirmed by the TEM (Fig. 2b and Fig. S1c, ESI $\dagger$ ) and dark field images (Fig. S1d, ESI $\dagger$ ). The morphology of CRDAs (Fig. 2c and Fig. S1e, f, ESI $\dagger$ ) was similar to that of DMSNs according to the microscopy images, illustrating that the loading of $\mathrm{Cu}_{2-x} \mathrm{~S}$ NPs did not alter the three-dimensional architecture of DMSNs. The loading mechanism of RB in CRDAs was based on the electrostatic attraction and pore encapsulations by DMSNs. Since RB is negatively charged, an electrostatic attraction between $\mathrm{RB}$ and host nanoparticles could facilitate the encapsulation of $\mathrm{RB}$ inside the pores of the $\mathrm{Cu}_{2-x} \mathrm{~S} @ D M S N-P E G-A E 105$ nanoparticles (Fig. S2, ESI $\dagger$ ). The pore diameter of DMSNs was calculated to be $21.5 \mathrm{~nm}$, determined via the $\mathrm{N}_{2}$ adsorption-desorption technique using Barrett-Joyner-Halenda methods (Fig. S3a, ESI $\dagger$ ). Comparatively, the specific surface area (SSA) of DMSNs and $\mathrm{Cu}_{2-x} \mathrm{~S} @ D M S N$ NPs are determined using the $\mathrm{N}_{2}$ adsorptiondesorption technique using Brunauer-Emmett-Teller methods (Fig. S3b, ESI $\dagger$ ). DMSNs and $\mathrm{Cu}_{2-x} \mathrm{~S} @ D M S N$ NPs showed specific surface areas (SSAs) of as high as $574.3 \mathrm{~m}^{2} \mathrm{~g}^{-1}$ and $482.7 \mathrm{~m}^{2} \mathrm{~g}^{-1}$, respectively, illustrating that ultrasmall $\mathrm{Cu}_{2-x} \mathrm{~S}$ NPs had been encapsulated inside the DMSNs nanoparticles. EDS (Fig. 2g) and corresponding EDS-mapping (Fig. 2h) exhibited uniformly distributed $\mathrm{Si}, \mathrm{O}, \mathrm{Cu}$, and $\mathrm{S}$ elements, illustrating the dispersive loading of $\mathrm{Cu}_{2-x} \mathrm{~S}$ NPs. The dynamic light scattering (DLS) data acquired in aqueous solutions demonstrate that the average hydrodynamic diameters $\left(D_{\mathrm{h}}\right)$ of DMSNs, and CRDAs were ca. 197 and $198 \mathrm{~nm}$ respectively (Fig. 2e). The XRD pattern of $\mathrm{Cu}_{2-x} \mathrm{~S}$ NPs most imitates the standard diffraction pattern of $\mathrm{Cu}_{5} \mathrm{~S}_{8}$ (Fig. S4, ESI $\dagger$ ). To further quantify the loading amounts of $\mathrm{Cu}_{2-x} \mathrm{~S}$ NPs and RB, inductively coupled plasma atomic emission spectrometry (ICP-AES) and UV-vis spectrometer were employed respectively. It has been determined that the encapsulation percentage of $\mathrm{Cu}_{2-x} \mathrm{~S}$ NPs (within CRDAs) is approximately $38.8 \%$, while that of RB sonosensitizer is ca. 8.2\% (within CRDAs) (Fig. S5a, b and S6, ESI $\dagger$ ). Although DMSNs can degrade faster than mesoporous silica of other configuration structures, it can maintain the configuration for several days in neutral and acidic environments. ${ }^{9}$ The release profile of $\mathrm{RB}$ from CRDAs under different $\mathrm{pH}$ conditions showed that $\mathrm{RB}$ can be released prior to the degradation of DMSNs (Fig. S7, ESI $\dagger$ ). The successful encapsulation of both
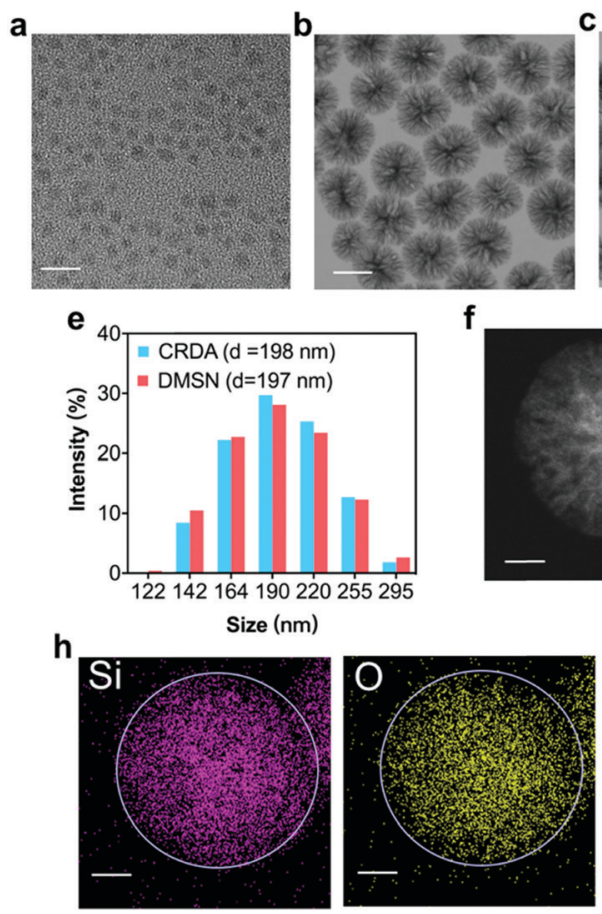

c

f
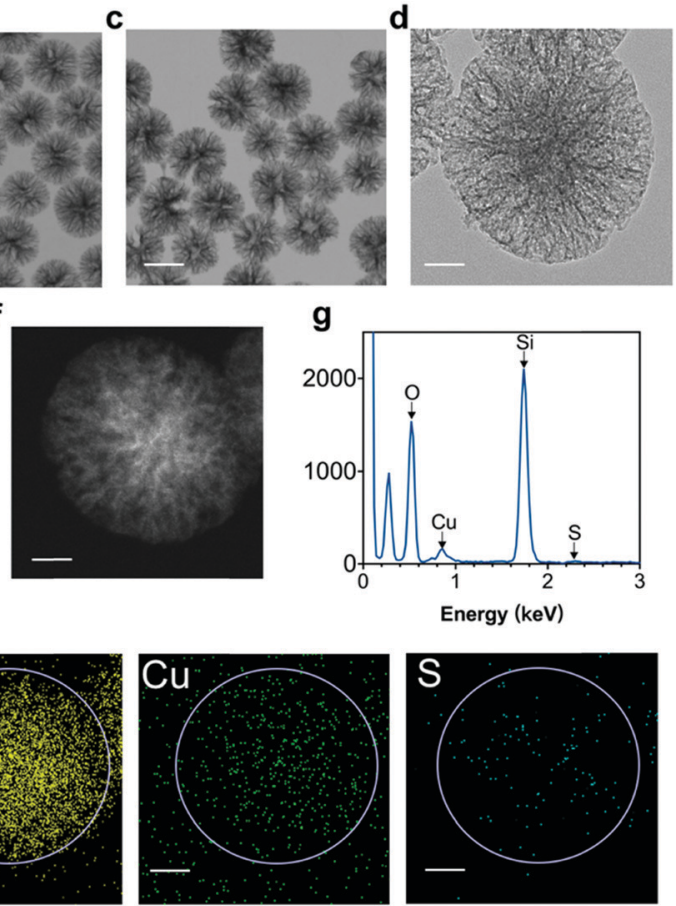

Fig. 2 Structural and composition characterizations of $\mathrm{Cu}_{2-x} \mathrm{~S} N P s$, DMSNs, CRDs and CRDAs. (a) TEM image of $\mathrm{Cu}_{2-x} \mathrm{~S} N P s$, (b) DMSNs and (c) CRDAs. (d) TEM image of CRDAs. (e) Size distribution profile of CRDAs and DMSNs measured by DLS. (f) Dark-field image (DFI), (g) energy dispersive spectrometry (EDS) and (h) corresponding elemental mapping (EDS-mapping) of CRDAs. Scale bar: (a), (d), (f) and (h): $20 \mathrm{~nm}$; (b) and (c): $100 \mathrm{~nm}$. 


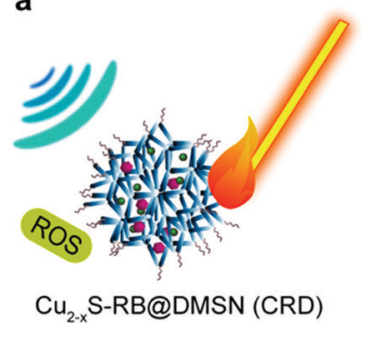

d

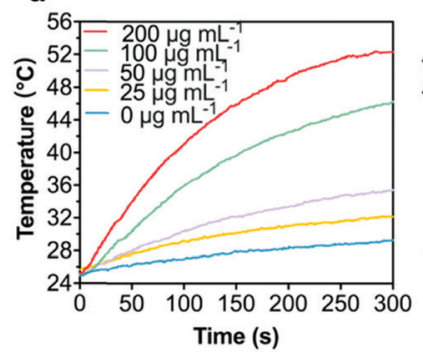

b

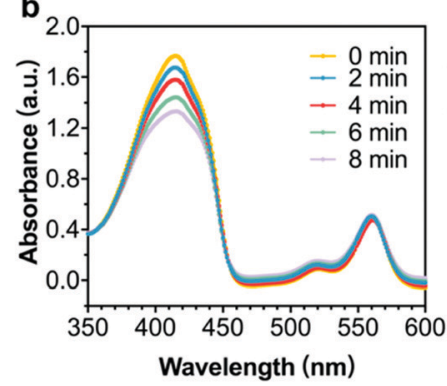

e

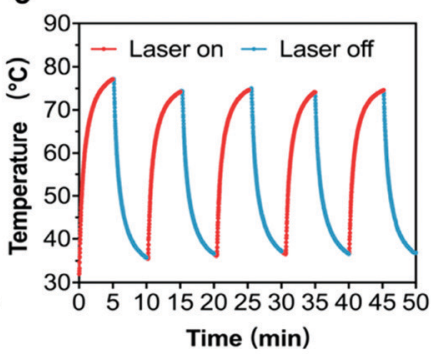

c
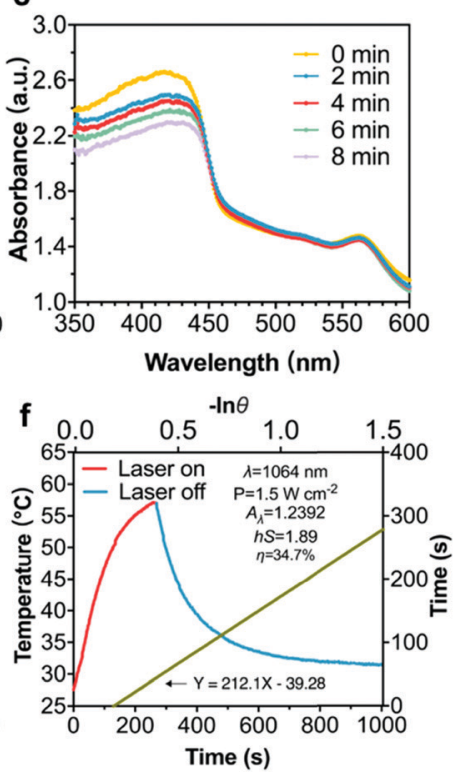

Fig. 3 Investigation of the PTT/SDT performance of CRDs. (a) Schematic illustration of synergistic PTT/SDT of CRDs. UV-vis absorption spectra of DPBF mixed with (b) RB and (c) CRDs after US irradiation ( $1 \mathrm{MHz}, 2.0 \mathrm{~W} \mathrm{~cm}^{-2}, 50 \%$ duty cycle) for $0,2,4,6,8$ min. (d) Localized temperature elevation curves of CRDs at a series of CRDs concentrations under the exposure to laser irradiation (1.5 W cm ${ }^{-2}$ ). (e) Photonic hyperthermia cycling profiles of CRDs during five sequential laser on and off cycles $\left(1.5 \mathrm{~W} \mathrm{~cm}^{-2}\right)$. (f) Specific period of heating and cooling curves of CRDs (250 $\mu \mathrm{g} \mathrm{mL} \mathrm{L}^{-1}$ ) and the linear fitting of time constants derived from the cooling curve.

photonic active $\mathrm{Cu}_{2-x} \mathrm{~S}$ NPs and sonodynamically active $\mathrm{RB}$ sonosensitizers into the DMSNs enables therapeutic purposes in the future.

\section{Sonodynamic and photonic hyperthermia performance of CRDs}

As stimulated by the US and/or laser irradiations, $\mathrm{Cu}_{2-x} \mathrm{~S}-\mathrm{RB} @$ DMSN NPs (CRDs) can enable ROS generation (via SDT process) and localized temperature elevation respectively (Fig. 3a) since SDT is essentially a sonosensitizer-mediated radical-generating process for tumor destruction upon US irradiation. The singlet oxygen $\left({ }^{1} \mathrm{O}_{2}\right)$ generation by $\mathrm{RB}$ sonosensitizers ${ }^{45}$ is the dominant factor to evaluate the sonodynamic performance of CRDs. Combined with ESR results (Fig. S8, ESI $\dagger$ ), it can be certified that CRDs as sonosensitizers can generate singlet oxygen $\left({ }^{1} \mathrm{O}_{2}\right)$ under US irradiation to induce the toxic effect and achieve the therapeutic function afterward. Therefore, 1,3-diphenylisobenzofuran (DPBF) was used exclusively to evaluate the generation level of ${ }^{1} \mathrm{O}_{2}$. The decline of the characteristic absorptions of DPBF was recorded via UV-vis spectrometry at the wavelength of $410 \mathrm{~nm}$ after US irradiation when DPBF mixtured sequentially with RB $\left(8 \mu \mathrm{g} \mathrm{mL}{ }^{-1}\right.$ ) (Fig. 3b) and CRDs ([RB] $=2 \mu \mathrm{g} \mathrm{mL} \mathrm{m}^{-1}$ ) (Fig. 3c) were subjected to the dark, indicating that CRDs with $\mathrm{RB}$ could efficiently induce ROS generation during the SDT process.

Photonic active $\mathrm{Cu}_{2-x} \mathrm{~S}$ NPs encapsulated inside DMSNs are capable of converting laser photon energy into heat. The hyperthermia performance of CRDs was assessed by the localized temperature elevation and photonic hyperthermia stability. Upon laser irradiation (1064 nm, $1.5 \mathrm{~W} \mathrm{~cm}^{-2}, 5 \mathrm{~min}$ ), the temperature of CRD aqueous suspension (at varied CRD concentrations: 25, 50, 100 and $200 \mu \mathrm{g} \mathrm{mL}^{-1}$ ) increased by 4 to 20 degrees in a dose-dependent manner, as compared with the control group (without CRDs) (Fig. 3d). In addition, the excellent photonic hyperthermia stability of CRDs was revealed by the negligible fluctuations of the maximum temperature between five sequential heating and cooling cycles (Fig. 3e). Finally, after detailed calculations, the photonic hyperthermalconversion efficiency $(\eta)$ of CRDs under $1064 \mathrm{~nm}$ laser was determined to be $34.7 \%$ according to the generally accepted calculation method ${ }^{18}$ (Fig. 3f).

\section{In vitro synergetic performance of PTT/SDT enabled by CRDs against OSC-19 cells}

According to the literature, the production of ROS and the generation of hyperthermia could kill cancer cells effectively (Fig. 4a). In order to evaluate the in vitro therapeutic performance against OSCC, we employed OSC-19 cells for CRDs coincubation. The cytotoxicity of CRDs was initially assessed by Cell Counting Kit-8 (CCK-8). According to the results, no obvious cytotoxicity was observed even when the concentration of CRDs reached as high as $800 \mu \mathrm{g} \mathrm{mL}{ }^{-1}$ after co-incubation with OSC-19 cells for 24 and $48 \mathrm{~h}$, indicating their high biocompatibility (Fig. 4b). Next, the therapeutic effects of SDT and PTT against CRDs-treated OSC-19 cells were estimated, irradiated with US and/or laser respectively (Fig. 4c-e). The cell viability of OSC-19 cells decreased along with the elevated concentration of CRDs after US and/or laser irradiation. At a dosage of $400 \mu \mathrm{g} \mathrm{mL}^{-1}$ of CRDs, the viabilities were $77.1 \%$ (US group), $45.9 \%$ (laser group), and $29.0 \%$ (US + laser group) respectively. According to in vitro cellular experiment, the 
a

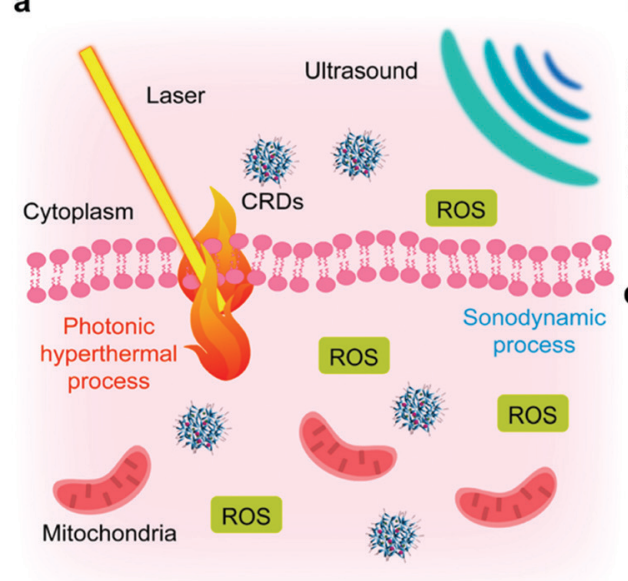

b
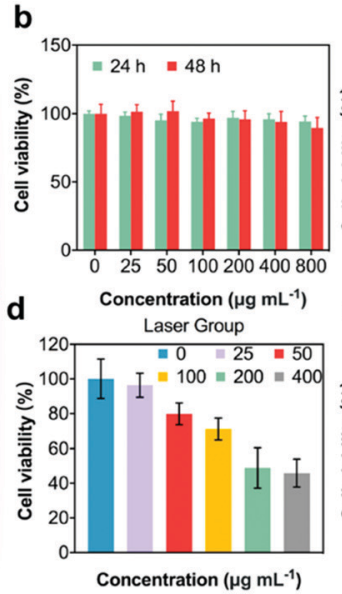

C US Group
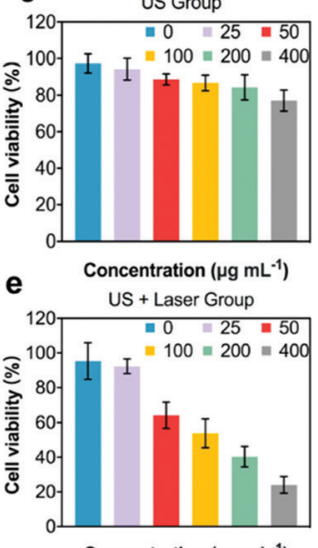

f Control
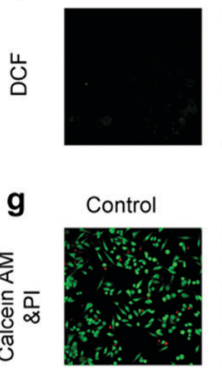

US

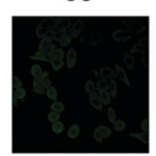

US

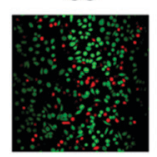

Laser

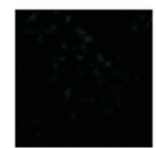

Laser

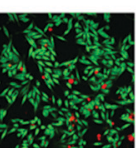

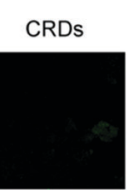

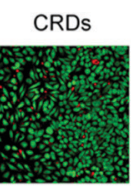

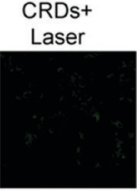

CRDs+
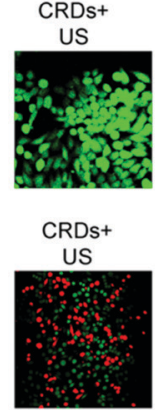

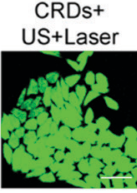

CRDs+ US+Laser

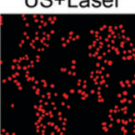

Fig. 4 In vitro cellular experiment of CRDs against OSC-19 cells. (a) Schematic diagram of the in vitro CRDs-based therapeutics against OSC-19 cells. Confocal images of OSC-19 cells stained with (f) DCFH-DA and (g) calcium-AM\&PI after respective treatments as indicated in the image. (b) Cell viabilities of OSC-19 cells after co-incubation with varied concentrations of CRDs for 24 and $48 \mathrm{~h}$. (c-e) Cell viabilities of OSC-19 cells co-incubated with varied concentrations of CRDs under US-only (US group) (c), laser-only (laser group) (d) and combinative US and laser (US + laser group) (e) treatments. Scale bar of (f and g): $50 \mu \mathrm{m}$.

dose-viability fitting curves of the three groups of cell therapy can reflect the therapeutic effect (Fig. S9, ESI $\dagger$ ). The decline in the curve of the US + laser group was significantly greater than that of the US group and the laser group. Therefore, combinative treatment of PTT/SDT against CRDs-treated OSC-19 cells induces the highest cytotoxicities, indicating that the synergetic SDT and PTT could improve the therapeutic efficacy over the respective monotherapy.

To visualize the SDT performance and therapeutic outcome, a DCFH-DA probe was used to indicate the cellular generation of ROS upon sonodynamic treatment, which is attributed to the transformation of non-fluorescent DCFH-DA to green fluorescent 2,7-dichlorofluorescein (DCF) in the presence of ROS. ${ }^{18}$ After co-incubating the cells with CRDs, DCFH-DA was supplemented to stain the cellular ROS for confocal observations. It has been observed that the cells in CRDs + US, CRDs + US + laser groups displayed the strongest green fluorescence signals, revealing the prominent generation of ROS (Fig. $4 \mathrm{f}$ and Fig. S10, $\mathrm{ESI} \dagger$ ). Comparatively, the cancer cells in other treatment groups exhibited weaker green fluorescence signals, implicating the inefficient ROS-generating capacity. To directly visualize the anti-tumor effects, calcein-AM and PI cell stains were supplemented after respective treatments (Fig. $4 \mathrm{~g}$ and Fig. S11, ESI $\dagger$ ). As indicated, a major proportion of dead cells (stained in red fluorescence) was observed in the synergetic CRDs + US + laser treatment group, as compared to that of the other treatment groups. Collectively, these experiments solidly validated the prominent synergetic therapeutic performance enabled by the in vitro PTT and SDT as enabled by the constructed CRDs.

\section{In vivo synergetic PTT/SDT cancer therapeutics enabled by CRDAs}

An in vivo biocompatibility assay was conducted to guarantee further in vivo therapeutics of CRDAs. Sixteen healthy BALB/c mice were randomly divided into four groups $(n=4$ in each group) followed by intravenous injection of CRDAs at elevated doses ( $0 \mathrm{mg} \mathrm{kg}^{-1}$ (as the control group), $5 \mathrm{mg} \mathrm{kg}^{-1}, 10 \mathrm{mg} \mathrm{kg}^{-1}$, and $\left.20 \mathrm{mg} \mathrm{kg}^{-1}\right)$. These mice were sacrificed at the end of the 15 day-evaluation period for harvesting the whole blood and major organs (i.e., heart, liver, spleen, lungs, and kidneys). As compared to the control group, the bodyweight profiles of mice between each group show no obvious variations during the therapeutics (Fig. 5a), and no significant variations in complete blood panel tests and biochemical analyses could be indicated (Fig. 5b). In addition, no substantial tissue damage or inflammatory lesions were discovered from hematoxylin and eosin (H\&E)-staining ultrathin sections of different major organs (Fig. 5c), suggesting that the injection of CRDAs (with a dose of as high as $20 \mathrm{mg} \mathrm{kg}^{-1}$ ) featured satisfactory in vivo 


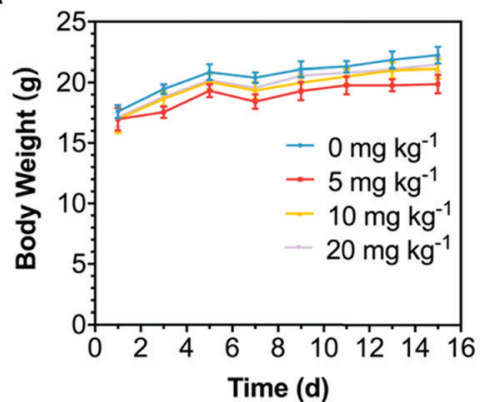

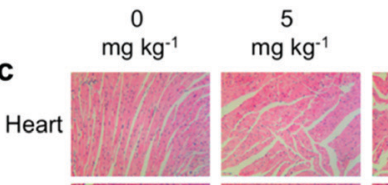
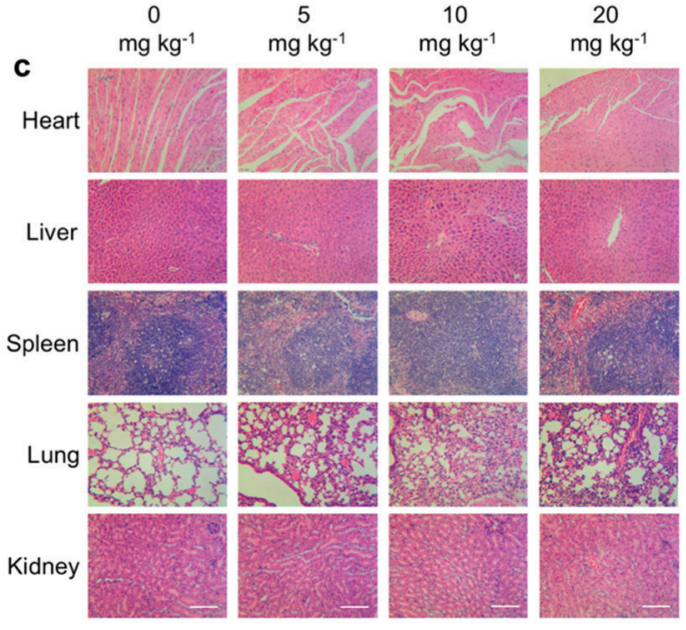

$-8$
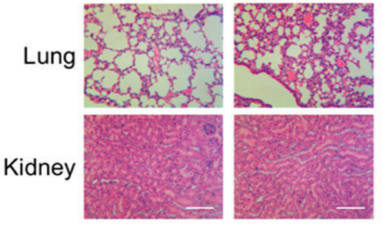

e

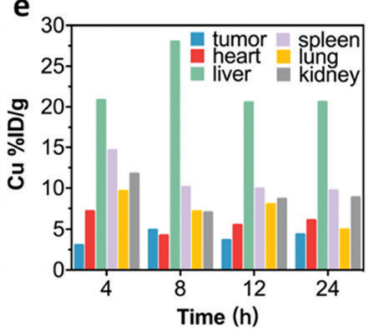

Fig. 5 In vivo biocompatibility assay and biodistribution of CRDAs. (a) Body weight of mice during 15 days in vivo biocompatibility evaluation experiments. (b) Whole blood panel tests and biochemical analyses. (c) H\&E-stained organ slices from mice after in vivo biocompatibility evaluation experiments. ( $d$ and e) In vivo biodistribution profiles of nontargeted CRDs (d) and targeted CRDAs (e) at different time-points post-injection. Scale bar of (c): $100 \mu \mathrm{m}$.

biocompatibility. To identify the in vivo targeting effects of CRDAs, the biodistribution of CRDAs was evaluated by analyzing the concentration of $\mathrm{Cu}$ species (Fig. $5 \mathrm{~d}$ and e) in the main organs and tumors at $4,8,12,24 \mathrm{~h}$ post-injection. The in vivo biodistribution evaluation suggests that CRDAs could effectively accumulate into tumor sites with a distribution dosage percentage of $10.99 \%$ (Fig. $5 \mathrm{~d}$ ) 4 h post-injection, which was significantly higher than that of CRDs without AE105 targeting design (3.03\%, Fig. 5e). Compared to nontargeting CRDs, the targeting CRDAs can accumulate higher and faster at the tumor site, while causing less pathological impact against other organs. In addition, the ICP-AES results showed that CRDAs and CRDs accumulated inside the tumor sites in $4 \mathrm{~h}$. By imaging the in vivo tumor sites via photoacoustics, ${ }^{27}$ targeting accumulation of CRDAs could also be indicated (Fig. S12a-c, ESI $\dagger$ ). The specifically enhanced accumulations of the nanoplatforms could further benefit the prominent therapeutics with mitigated cytotoxicity.

The in vitro synergetic PTT and SDT by CRDs and their desirable in vivo biocompatibility encourage further systematic in vivo therapeutics of CRDAs against OSC-19 cells. During the investigations, forty-two BALB/c nude mice were randomly divided into seven groups ( $n=6$ in each group) prior to the tumor xenograft establishment. When the volume of the tumor xenografts reached $50 \mathrm{~mm}^{3}$, these nude mice were injected with saline (for mice in control, US, laser groups) and CRDAs (for mice in CRDAs, CRDAs + US, CRDAs + laser, CRDAs + US + laser groups) respectively. CRDA-treated mice were further exposed to external stimuli irradiation including US and/or laser correspondingly (Fig. 6a). During NIR-laser irradiation (for CRDAs + US + laser group), the localized temperature of tumor sites increased from $24{ }^{\circ} \mathrm{C}$ to $54{ }^{\circ} \mathrm{C}$ while for mice in the laser group, merely 5 degrees of temperature increase was recorded (Fig. 6b). During the 15 day-therapeutics, the body weight of mice and the dimension of the tumors were recorded every other day. The body weight profiles of mice between each group show no obvious variations during the therapeutics, implying the biocompatibility of the therapeutic modalities (Fig. 6c). The tumor xenografts of mice in control, US, laser and CRDAs groups grew extensively within 15 days, while mice receiving synergistic PTT/SDT treatments enabled by CRDAs in CRDAs + US + laser group exhibited intense therapeutic efficacy with an inhibition rate (IR) of $103.4 \%$ (meaning destruction rather than inhibition), significantly higher than that of the CRDAs + US group (49.79\%) and CRDAs + laser group (61.89\%) (Fig. 6d), verifying the effectiveness of the synergetic therapeutic consequence of PTT/SDT enabled by the targeting CRDAs.

At $24 \mathrm{~h}$ post-treatments, one tumor-bearing mouse from each group was euthanized and the major organs and tumors were dissected for detailed pathological analyses by H\&E, TdTmediated dUTP Nick-End Labeling (TUNEL) and antigen Ki-67 immunostaining respectively (Fig. 6e). As compared to the control, US, laser and CRDAs groups, mice in the monotherapeutic 

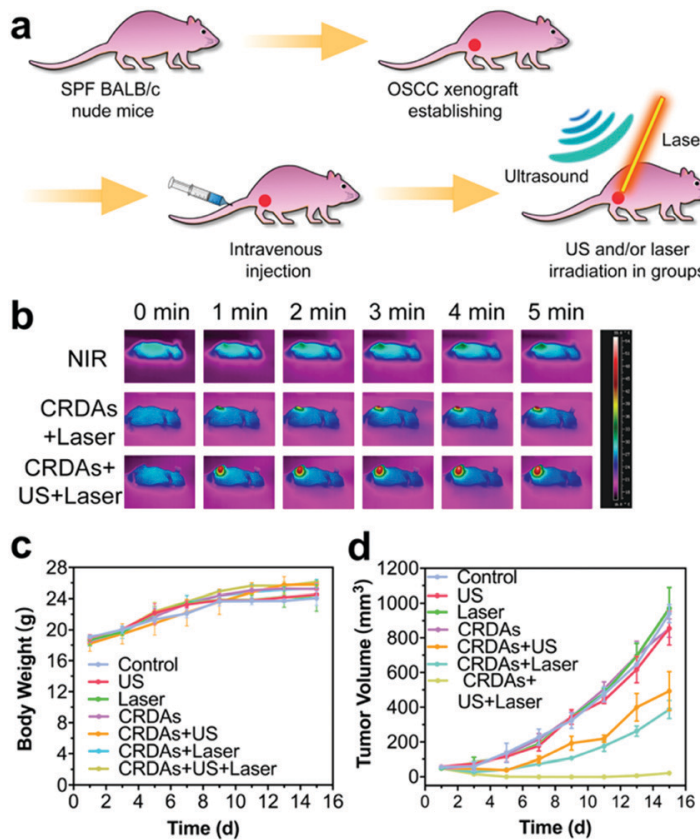

\section{$\mathbf{e}$ \\ Control \\ us}
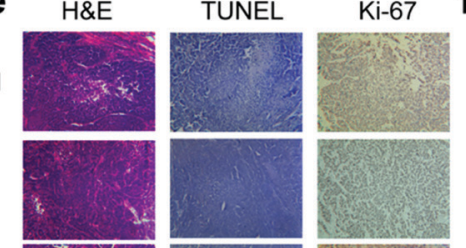

Laser
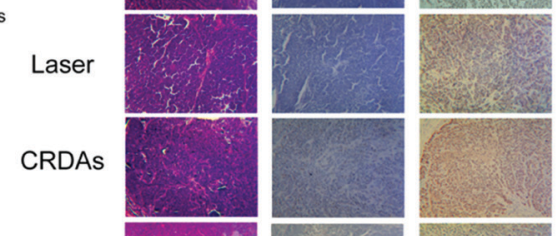

\section{CRDAs} + US

CRDAs +Laser

CRDAs+ US+Laser
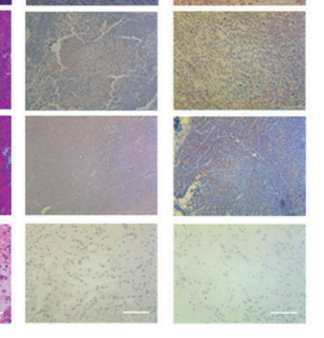

Digital Photograph

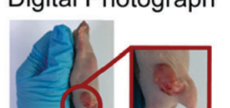

Fig. 6 In vivo antitumor experiments of CRDAs against OSCC tumor xenografts. (a) Schematic schedule diagram of the in vivo synergetic PTT/SDT therapeutics. (b) Infrared thermal images of mice during PTT treatment along the irradiation time. (c) Body weight profile and (d) tumor volume profile of mice in each group during the in vivo therapeutic treatment period. (e) Histological microscopic images of tumor sections stained with H\&E, TUNEL and antigen $\mathrm{Ki}-67$ of mice. (f) Digital photographs of tumors of mice in each group collected at the end of the in vivo therapeutic experiment. Scale bar of (e): $100 \mu \mathrm{m}$.

CRDAs + US and CRDAs + laser groups exhibited reduction of the tumor cells, enlarged proportions of the apoptotic area (in H\&E and TUNEL staining microscopy images), and mitigated expressions of the cell proliferation (in antigen Ki-67 staining microscopy images). Comparatively, the synergetic PTT/SDT therapy was conceived to deliver much prominent consequence. The xenografts of mice in the CRDAs + US + laser group were almost eradicated, as revealed by, H\&E, TUNEL, antigen Ki-67 staining images and the digital photographs (Fig. 6f). The survival rate suggested that photonic hyperthermal and sonodynamic targeting therapy can extend the living time of mice (Fig. S13, ESI $\dagger$ ). These results collectively demonstrate the prominent therapeutic effects of the synergetic PTT/SDT therapeutics enabled by CRDAs against OSCC with satisfactory biocompatibility.

\section{Conclusions}

In summary, we have constructed an intriguing uPAR-targeting ligand AE105-decorated DMSNs-based delivery system co-encapsulated with photonic active $\mathrm{Cu}_{2-x} \mathrm{~S}$ nanoagents and sonosensitizer RB (abbreviated as CRDAs) for high-performance OSCCtargeting and synergetic PTT/SDT nanotherapeutics. Based on the prominent hyperthermia and sonodynamic performance of the integrated $\mathrm{Cu}_{2-x} \mathrm{~S}$ NPs and RB respectively, the rationally engineered CRDAs could efficiently enable satisfactory tumor destruction against the OSCC both in vitro and in vivo. Featuring the targeting performance of AE105, the enhanced accumulation of CRDAs into tumor xenografts was achieved on an in vivo OSCC tumor xenograft. Together with the high biocompatibility of the designed CRDAs, a prominent tumor inhibition rate of $103.4 \%$ was realized. The present synergetic strategy has illustrated a satisfactory therapeutic modality to treat oral squamous cell carcinoma with both effectiveness and biocompatibility.

\section{Conflicts of interest}

There are no conflicts to declare.

\section{Acknowledgements}

We greatly acknowledge the financial support from the National Natural Science Foundation of China (Grant No. 81971618, 51672303, and 51722211), National Key R\&D Program of China (Grant No. 2016YFA0203700), Program of Shanghai Subject Chief Scientist (Grant No. 18XD1404300) and China Postdoctoral Science Foundation (Grant No. 2020M671243).

\section{Notes and references}

1 S. Monro, K. L. Colon, H. Yin, J. Roque, 3rd, P. Konda, S. Gujar, R. P. Thummel, L. Lilge, C. G. Cameron and S. A. McFarland, Chem. Rev., 2019, 119, 797-828.

2 H. Wu, L. Song, L. Chen, W. Zhang, Y. Chen, F. Zang, H. Chen, M. Ma, N. Gu and Y. Zhang, Acta Biomater., 2018, 74, 302-311.

3 W. Yue, L. Chen, L. Yu, B. Zhou, H. Yin, W. Ren, C. Liu, L. Guo, Y. Zhang, L. Sun, K. Zhang, H. Xu and Y. Chen, Nat. Commun., 2019, 10, 2025. 
4 M. Zhao, Y. Xu, M. Xie, L. Zou, Z. Wang, S. Liu and Q. Zhao, Adv. Healthcare Mater., 2018, 7, e1800606.

5 Z. Zhao, K. Xu, C. Fu, H. Liu, M. Lei, J. Bao, A. Fu, Y. Yu and W. Zhang, Biomaterials, 2019, 219, 119379.

6 Z. Jin, K. Wu, J. Hou, K. Yu, Y. Shen and S. Guo, Biomaterials, 2018, 153, 49-58.

7 F. Gao, G. He, H. Yin, J. Chen, Y. Liu, C. Lan, S. Zhang and B. Yang, Nanoscale, 2019, 11, 2374-2384.

8 J. Shi, Z. Chen, B. Wang, L. Wang, T. Lu and Z. Zhang, ACS Appl. Mater. Interfaces, 2015, 7, 28554-28565.

9 M. Huo, L. Wang, Y. Chen and J. Shi, Nat. Commun., 2017, 8, 357.

10 T. Suteewong, H. Sai, R. Hovden, D. Muller, M. S. Bradbury, S. M. Gruner and U. Wiesner, Science, 2013, 340, 337-341.

11 F. Tang, L. Li and D. Chen, Adv. Mater., 2012, 24, 1504-1534.

12 Y. Wang, Q. Zhao, N. Han, L. Bai, J. Li, J. Liu, E. Che, L. Hu, Q. Zhang, T. Jiang and S. Wang, Nanomedicine, 2015, 11, 313-327.

13 C. M. Carnielli, C. C. S. Macedo, T. De Rossi, D. C. Granato, C. Rivera, R. R. Domingues, B. A. Pauletti, S. Yokoo, H. Heberle, A. F. Busso-Lopes, N. K. Cervigne, I. SawazakiCalone, G. V. Meirelles, F. A. Marchi, G. P. Telles, R. Minghim, A. C. P. Ribeiro, T. B. Brandao, G. de Castro, Jr., W. A. Gonzalez-Arriagada, A. Gomes, F. Penteado, A. R. Santos-Silva, M. A. Lopes, P. C. Rodrigues, E. Sundquist, T. Salo, S. D. da Silva, M. A. Alaoui-Jamali, E. Graner, J. W. Fox, R. D. Coletta and A. F. Paes Leme, Nat. Commun., 2018, 9, 3598.

14 R. L. Siegel, K. D. Miller and A. Jemal, Ca-Cancer J. Clin., 2016, 66, 7-30.

15 D. A. Mahvi, R. Liu, M. W. Grinstaff, Y. L. Colson and C. P. Raut, Ca-Cancer J. Clin., 2018, 68, 488-505.

16 S. R. Gvetadze, P. Xiong, M. Lv, J. Li, J. Hu, K. D. Ilkaev, X. Yang and J. Sun, Dentomaxillofac. Radiol., 2017, 46, 20160345.

17 J. A. Ochoa-Alvarez, H. Krishnan, J. G. Pastorino, E. Nevel, D. Kephart, J. J. Lee, E. P. Retzbach, Y. Shen, M. Fatahzadeh, S. Baredes, E. Kalyoussef, M. Honma, M. E. Adelson, M. K. Kaneko, Y. Kato, M. A. Young, L. Deluca-Rapone, A. J. Shienbaum, K. Yin, L. D. Jensen and G. S. Goldberg, Oncotarget, 2015, 6, 9045-9060.

18 C. Dai, S. Zhang, Z. Liu, R. Wu and Y. Chen, ACS Nano, 2017, 11, 9467-9480.

19 H. Lin, W. Qiu, J. Liu, L. Yu, S. Gao, H. Yao, Y. Chen and J. Shi, Adv. Mater., 2019, 31, e1903013.

20 X. Wang, Z. Geng, H. Cong, Y. Shen and B. Yu, ChemBioChem, 2019, 20, 1628-1636.

21 Y. Cao, S. Li, C. Chen, D. Wang, T. Wu, H. Dong and X. Zhang, Biomaterials, 2018, 158, 23-33.

22 R. He, Y. C. Wang, X. Wang, Z. Wang, G. Liu, W. Zhou, L. Wen, Q. Li, X. Wang, X. Chen, J. Zeng and J. G. Hou, Nat. Commun., 2014, 5, 4327.
23 W. Xu, J. Qian, G. Hou, A. Suo, Y. Wang, J. Wang, T. Sun, M. Yang, X. Wan and Y. Yao, ACS Appl. Mater. Interfaces, 2017, 9, 36533-36547.

24 W. Gao, Y. Sun, M. Cai, Y. Zhao, W. Cao, Z. Liu, G. Cui and B. Tang, Nat. Commun., 2018, 9, 231.

25 Q. L. Li, Y. Sun, L. Ren, X. Wang, C. Wang, L. Li, Y. W. Yang, $\mathrm{X}$. Yu and J. Yu, ACS Appl. Mater. Interfaces, 2018, 10, 29314-29324.

26 R. Ge, C. Liu, X. Zhang, W. Wang, B. Li, J. Liu, Y. Liu, H. Sun, D. Zhang, Y. Hou, H. Zhang and B. Yang, ACS Appl. Mater. Interfaces, 2018, 10, 20342-20355.

27 J. Mou, P. Li, C. Liu, H. Xu, L. Song, J. Wang, K. Zhang, Y. Chen, J. Shi and H. Chen, Small, 2015, 11, 2275-2283.

28 Z. Zhou, Y. Yan, K. Hu, Y. Zou, Y. Li, R. Ma, Q. Zhang and Y. Cheng, Biomaterials, 2017, 141, 116-124.

29 X. Qian, Y. Zheng and Y. Chen, Adv. Mater., 2016, 28, 8097-8129. 30 J. Nam, S. Son, L. J. Ochyl, R. Kuai, A. Schwendeman and J. J. Moon, Nat. Commun., 2018, 9, 1074.

31 Y. Wang, W. Zhang, P. Sun, Y. Cai, W. Xu, Q. Fan, Q. Hu and W. Han, Theranostics, 2019, 9, 391-404.

32 S. D. da Silva, M. Hier, A. Mlynarek, L. P. Kowalski and M. A. Alaoui-Jamali, Front. Pharmacol., 2012, 3, 149.

33 L. A. Garraway and P. A. Janne, Cancer Discovery, 2012, 2, 214-226.

34 B. Yang, Y. Chen and J. Shi, Chem. Rev., 2019, 119, 4881-4985. 35 C. McEwan, C. Fowley, N. Nomikou, B. McCaughan, A. P. McHale and J. F. Callan, Langmuir, 2014, 30, 14926-14930.

36 C. McEwan, J. Owen, E. Stride, C. Fowley, H. Nesbitt, D. Cochrane, C. C. Coussios, M. Borden, N. Nomikou, A. P. McHale and J. F. Callan, J. Controlled Release, 2015, 203, 51-56.

37 C. R. Leemans, B. J. Braakhuis and R. H. Brakenhoff, Nat. Rev. Cancer, 2011, 11, 9-22.

38 F. Blasi and P. Carmeliet, Nat. Rev. Mol. Cell Biol., 2002, 3, 932-943.

39 M. A. Pavon, I. Arroyo-Solera, M. V. Cespedes, I. Casanova, X. Leon and R. Mangues, Oncotarget, 2016, 7, 57351-57366.

40 H. W. Smith and C. J. Marshall, Nat. Rev. Mol. Cell Biol., 2010, 11, 23-36.

41 Y. W. Chen, T. Y. Liu, P. H. Chang, P. H. Hsu, H. L. Liu, H. C. Lin and S. Y. Chen, Nanoscale, 2016, 8, 12648-12657.

42 A. Christensen, K. Juhl, M. Persson, B. W. Charabi, J. Mortensen, K. Kiss, G. Lelkaitis, N. Rubek, C. von Buchwald and A. Kjaer, Oncotarget, 2017, 8, 15407-15419.

43 M. C. Kriegbaum, M. Persson, L. Haldager, W. AlpizarAlpizar, B. Jacobsen, H. Gardsvoll, A. Kjaer and M. Ploug, Curr. Drug Targets, 2011, 12, 1711-1728.

44 M. Persson, M. Hosseini, J. Madsen, T. J. Jorgensen, K. J. Jensen, A. Kjaer and M. Ploug, Theranostics, 2013, 3, 618-632.

45 B. McCaughan, C. Rouanet, C. Fowley, N. Nomikou, A. P. McHale, P. A. McCarron and J. F. Callan, Bioorg. Med. Chem. Lett., 2011, 21, 5750-5752. 